\title{
Multiagent and Particle Swarm Optimization for Ship Integrated Power System Network Reconfiguration
}

\author{
Zheng Wang, ${ }^{1,2}$ Li Xia, ${ }^{2}$ Yongji Wang, ${ }^{1}$ and Lei Liu ${ }^{1}$ \\ ${ }^{1}$ School of Automation, Huazhong University of Science and Technology, Wuhan 430074, China \\ ${ }^{2}$ School of Electrical Engineering, Naval University of Engineering, Wuhan 430033, China \\ Correspondence should be addressed to Yongji Wang; wangyjch@mail.hust.edu.cn
}

Received 11 December 2013; Accepted 2 February 2014; Published 25 March 2014

Academic Editor: Huaicheng Yan

Copyright (c) 2014 Zheng Wang et al. This is an open access article distributed under the Creative Commons Attribution License, which permits unrestricted use, distribution, and reproduction in any medium, provided the original work is properly cited.

\begin{abstract}
Ship integrated power system adopts electric power propulsion. Power network and electric power network are integrated into complicated one. Network reconfiguration of ship integrated power system is a typical nonlinear optimization that is multitarget and multiconstraint. According to the characteristics of ship integrated power system, simplified network model and reconfiguration mathematical model are established. A multiagent and particle swarm optimization is presented to solve network reconfiguration problem. The results of simulation show that multiagent and particle swarm optimization can reconfigure ship integrated power system efficiently.
\end{abstract}

\section{Introduction}

Ship integrated power system (SIPS) adopts electric power propulsion. Power network and electric power network are integrated into complicated one. Integrated power system is the trend of ship power system. Due to the application of high power density-integrated generating system, DC medium voltage transmission, zonal distribution system, power conversion device and high power electric propulsion system, and the generation capacity and scale of SIPS keep on enlarging. The way the system works and its protection become more complex. It is important to reconfigure the system as fast as possible when faults occur. Because of many differences between the ship and the land power system, the network reconfiguration of land power system which is based on how to lower the network loss of system does not work well on the ship [1-5]. Network reconfiguration of SIPS is to be researched.

Network reconfiguration of SIPS is a nonlinear combinatorial optimization problem. Srivastava et al. used improved algorithm for reconfiguration, but it cannot restore the loads as much as possible [6]; Nagata used multiagent technique for reconfiguration, but it also cannot make the result most optimal [7].
Based on analyzing many methods in existence and according to the characteristics of ship integrated power system, a multiagent and particle swarm optimization (MAPSO) is presented to solve the power system network reconfiguration problem. Theoretical analysis and simulation results show that MAPSO can reconfigure the network of ship integrated power system better.

\section{Simplified Network Model and Reconfiguration Mathematical Model of SIPS}

2.1. Simplified Network Model of SIPS. A typical SIPS is made up of 4 power stations. There are 2 generators in each station. Power stations connect with each other by jumper wires. Generators generate DC $4000 \mathrm{~V}$ power, which is converted to DC $700 \mathrm{~V}$ power by converters and access to DC zonal distribution switchboards and then to AC $400 \mathrm{~V}$ by inverters or DC $230 \mathrm{~V}$ by choppers. Loads in SIPS include propulsion loads, important loads, and common loads. According to the importance, the loads can be graded into three ranks, of which Rank 1 and Rank 2 loads usually have two power supply routes, the normal one and the alternative. When 


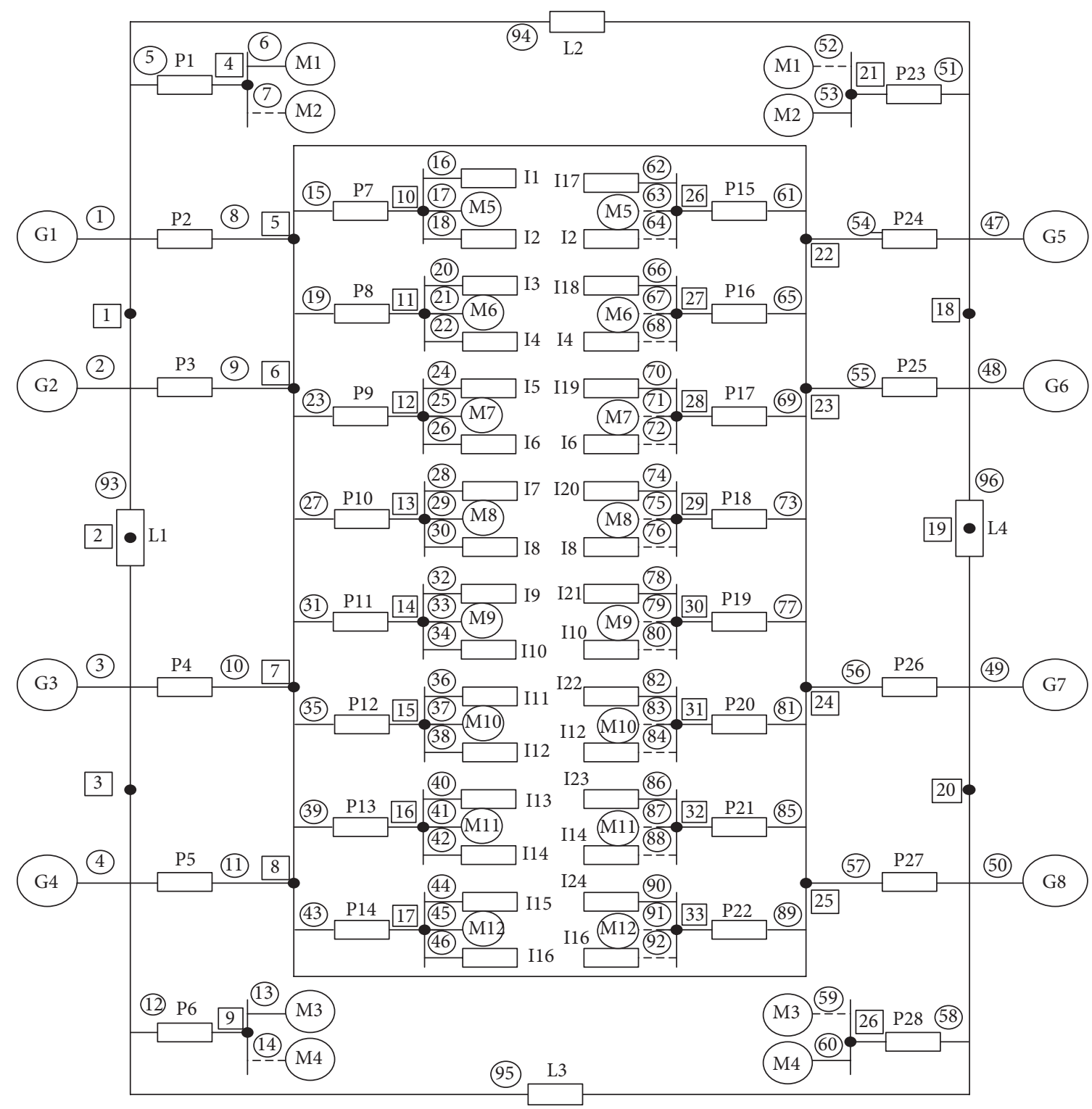

FIGURE 1: The simplified network model of SIPS.

the SIPS goes wrong, the circuit breaker or other protective devices isolate the failure load or generator, by changing the state of the switch to ensure that the important load can get maximum degree of power restoration under the system constraints, and unimportant loads are removed if necessary.

According to the graph theory [8-10], the electrical devices (including feeders and jumper wires connected with the device) in SIPS are abstracted to be branches, while the connection points between the devices are abstracted to be nodes. Then, we can establish a node-branch topology mode. Depth-first search and breadth-first search are adopted to number the branches and nodes. First, we should number generators branches by breadth-first search. Then, we number distribution branches also by breadth-first search. If we meet feeder branches, we adopt depth-first search for numbering, until we meet load branches. After that, we number the jumper wires.
The simplified network model of SIPS is shown in Figure 1. (1)-6) represents braches, $1-33$ represent nodes, G1-G8 are generators, M1-M4 are propulsion loads, M5M12 are motor loads, I represents static loads, P1-P28 are power electronic equipment, and L1-L4 are jumper wires. The alternative routes of propulsion and important loads are connected by dashed lines.

2.2. Network Reconfiguration Mathematical Model. SIPS requires the maximum loads to restore and supply power for important loads (Rank 1 and Rank 2) in priority after network reconfiguration and make the operation times of switches as few as possible [11]. So the object function can be expressed as follows:

$$
\max F(X)=\sum_{i=1}^{n_{1}} \lambda_{1} L_{g 1 i}+\sum_{i=1}^{n_{2}} \lambda_{2} L_{g 2 i}+\sum_{i=1}^{n_{3}} \lambda_{3} L_{g 3 i}-\eta S_{x},
$$


where $F(X)$ is the total power restored; $L_{g 1 i}, L_{g 2 i}$, and $L_{g 3 i}$ are the 3 grade loads, $n_{1}, n_{2}$, and $n_{3}$ are the total number of each grade loads, $\lambda_{1}, \lambda_{2}$, and $\lambda_{3}$ are the weight coefficients of each grade loads, $S_{x}$ is the total operation times of switches, and $\eta$ is the punitive weight coefficient. We can get the maximum important load restored and the least operation times of switches by choosing the proper weight coefficients. For example, $\lambda_{1}=1$ for Rank 1 loads, $\lambda_{2}=0.1$ for Rank 2 loads, and $\lambda_{3}=0.01$ for Rank 3 loads.

The network reconfiguration of SIPS should be constrained as follows.

(1) Power flow constraint: $A P=D$, where $A$ is the node-branch incidence matrix, $P$ is thefeeder current vector, and $D$ is the load demand vector.

(2) Capacity constraint: $P_{i} \leq P_{i}^{\max }$.

(3) Voltage constraint: $V_{i \min } \leq V \leq V_{i \max }$.

(4) Radiation constraint: there is only one route connected with the load restored, normal one or alternative.

(5) Priority constraint: priority of power supply is based on the ranks of loads.

\section{Multiagent and Particle Swarm Optimization}

3.1. Application of Multiagent in Network Reconfiguration of SIPS. Generally, agent is an entity with active behavioral capacity in any environment, such as organism, software system or controller in control system. Multiagent system (MAS) is a loose coupling network formed by several agents. Physically or logically the agents are scattered, and their behaviors are self-governed. That is to say, their targets and behaviors would not be restricted by any other agents. To achieve the same task or the same goal, all the agents link with each other under some kind of protocol. They can solve problems beyond single agent's capability by communication and cooperation [12-14].

Considering regional autonomy of multiagent, the set of nonswitch devices controlled by switch devices on the same regional feeder (including generators, loads, jumper wires, switches, etc.) can be regarded as one agent. Therefore, the SIPS network may be divided into some separate power supply agents.

The SIPS network shown in Figure 1 can be divided into several feeder units. Each one is abstracted as a regional feeder agent. A regional feeder multiagent network model is proposed, as shown in Figure 2.

The regional feeder agents are intelligent; they can judge and make decision autonomous. According to the operation condition of SIPS, the regional feeder agents can communicate electric parameters, such as power, current, and voltage, with adjacent agents so as to guarantee stable operation of SIPS.

3.2. Particle Swarm Optimization. In 1995, the American social psychologist James Kennedy and electrical engineers

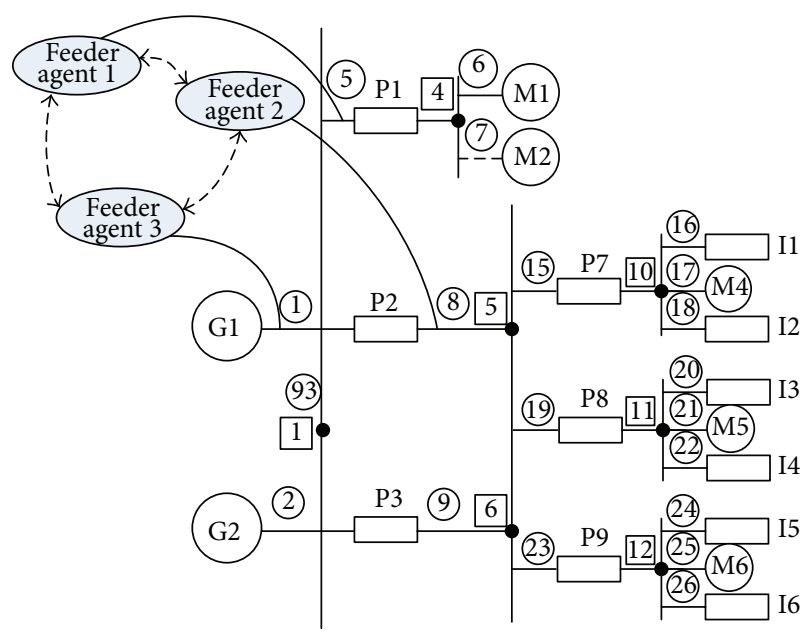

FIGURE 2: Regional feeder multiagent network model of SIPS.

Russell Eberhart proposed the particle swarm algorithm [15]. The positions of particles are used to solve the optimization problem. In the algorithm, a velocity vector of a particle determines the flight direction and speed. Located in an $M$ dimensional search space, the location of particle $i$ can be expressed as $X_{i}=\left(x_{i 1}, x_{i 2}, \ldots, x_{i D}\right)^{T}$, and speed can be expressed as $V_{i}=\left(v_{i 1}, v_{i 2}, \ldots, v_{i D}\right)^{T}$. The best position of the particle is $X_{i}^{p b}=\left(X_{i 1}^{p b}, X_{i 2}^{p b}, \ldots, X_{i D}^{p b}\right)^{T}$, while the best position of the whole swarm is $X^{g b}=\left(X_{1}^{g b}, X_{2}^{g b}, \ldots, X_{D}^{g b}\right)^{T}$. In the process of swarm initialization, the particles are distributed in the whole solution space randomly, to obtain the optimal solution through iteration gradually. Each particle tracks $X^{p b}$ and $X^{g b}$ to determine their movement [16-22]:

$$
\begin{gathered}
V_{i d}^{t+1}=\omega V_{i d}^{t}+c_{1} R\left(X_{i d}^{p b}-X_{i d}^{t}\right)+c_{2} R\left(X_{d}^{g b}-X_{i d}^{t}\right), \\
X_{i d}^{t+1}=X_{i d}^{t}+V_{i d}^{t+1},
\end{gathered}
$$

where $d=1,2, \ldots, D, D$ is the particle dimension and $t$ is iterations. $\omega$ is inertia weight, $c_{1}$ and $c_{2}$ are the cognitive and social parameters, and $R$ is a random number between 0 and 1 . In addition, the velocity of particles $V_{i d}$ is limited by a maximum speed $V_{\text {max }, d}$.

Consider that the position of the particles in each dimension and the best place of individual particle are 0 or 1 . Then, only the particle speed is considered, and introduce the speed formula, that is, sigmoid function as follows:

$$
\operatorname{Sigmoid}(x)=\frac{1}{1+e^{-x}} .
$$

The bigger the speed value of particle is, the closer the sigmoid function value is to 1 . However, the smaller the speed value is, the closer the sigmoid function is to 0 . So the value of sigmoid function can be considered as the probability of the particle position, that is, 0 or 1 . 
Meanwhile, considering the sigmoid function is not saturated, it is to make the following adjustment:

$$
\text { Sigmoid }(x)= \begin{cases}0.98, & x>4 \\ \frac{1}{1+e^{-x}}, & -4 \leq x \leq 4 \\ 0.02, & x<4 .\end{cases}
$$

In the iterative process, the calculation formula of $X_{i d}^{t+1}$ is

$$
X_{i d}^{t+1}= \begin{cases}0, & \text { rand } \geq \operatorname{Sigmoid}\left(V_{i d}^{t+1}\right) \\ 1, & \text { rand }<\operatorname{Sigmoid}\left(V_{i d}^{t+1}\right),\end{cases}
$$

where rand is the random number in the interval $[0,1]$.

3.3. Multiagent and Particle Swarm Optimization. MAPSO is an intelligent algorithm based on PSO and multiagent with autonomous learning, competition, and collaboration. Regional feeder agents are defined by the SIPS network model. The agents learn evolutional mechanism of PSO by self-learning and communication with adjacent agents and update the particle swarm according to some rules, so as to make the swarm converge to the global optimal solution faster and more accurate.

In MAPSO, a regional feeder agent is equal to a particle in the particle swarm, so that the topology of the particle swarm is determined by the SIPS network reconfiguration model. By agents' self-updating and cooperation with adjacent agents, MAPSO can present a solution for the SIPS network reconfiguration. Multiagent can get the optimal objective function value in PSO. The flow chart of SIPS network reconfiguration based on MAPSO is shown in Figure 3.

\section{Simulation Results}

For the SIPS network model in Figure 1, according to the different complexity degrees of faults, there are two cases of reconfiguration using MAPSO.

The data sources of samples are based on the simplified network model of SIPS in Figure 1. Power of devices is changed into standard per unit, as shown in Table 1.

In the simulation, particles of swarm represent the states of branches in the power system, 0 for circuit open and 1 for circuit closed.

Example 1. Assuming that a fault occurs in regional feeder agent 17, MAPSO is used to carry out network reconfiguration, and simulation results are as follows.

Regional feeder agent 63: the initial value is 0 ; the ultimate value is 1 .

Fitness value is 5.63305 and switch operation times (SOT) is is 1 .

The changes of fitness and SOT are shown in Figure 4. Simulation results show that regional feeder agent 17 communicates with adjacent agent when fault occurs, and then

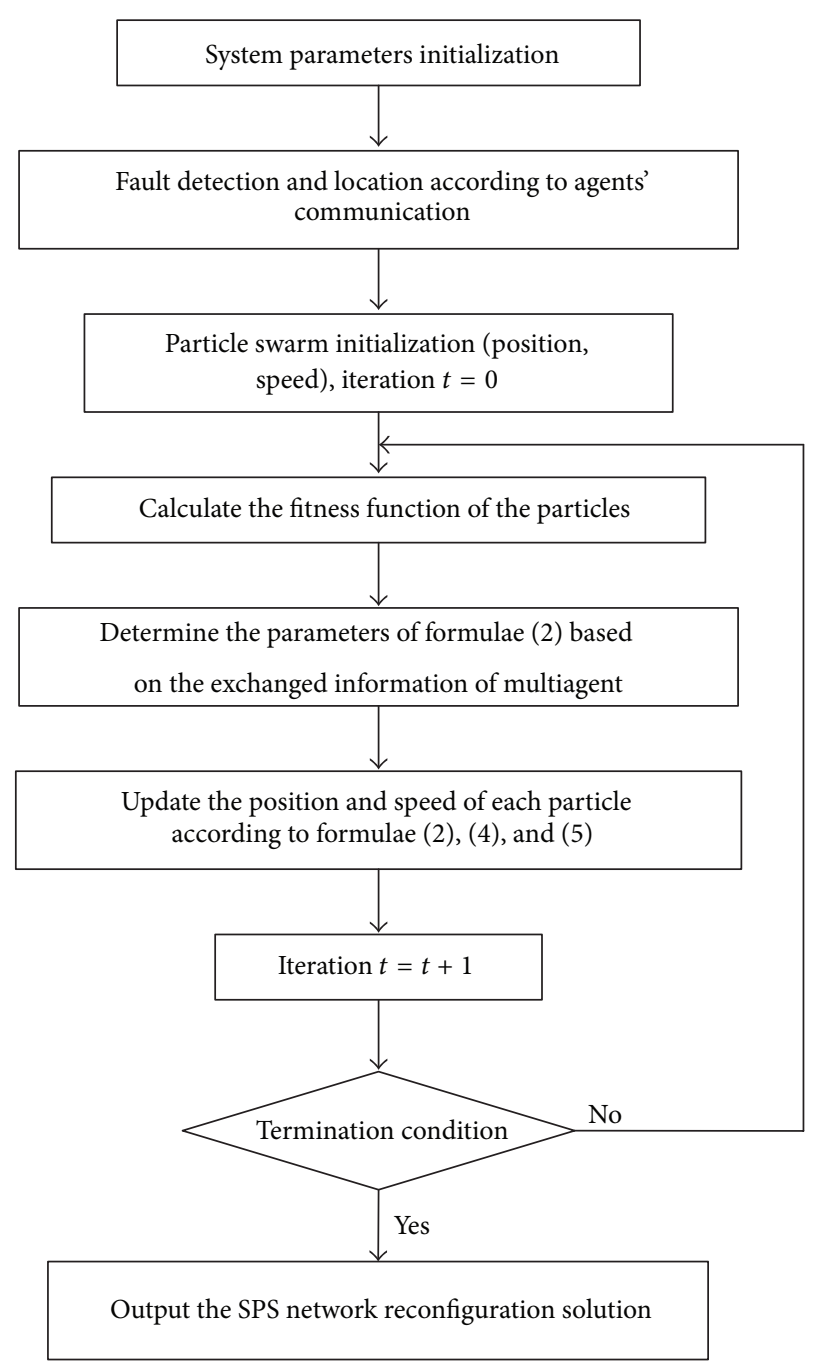

FIGURE 3: The flow chart of SIPS network reconfiguration based on MAPSO.

chooses the alternative route (regional feeder agent 63) for power supply. MAPSO gets the best fitness value 5.63305 from the 16th iteration, but only until the 18th iteration it gets the minimum SOT: 1 . So we get the best network reconfiguration solution from the 18 th iteration.

Example 2. Assuming that faults occur in regional feeder agents 3, 31, 93, and 95, MAPSO is used to carry out network reconfiguration, and simulation results are as follows.

Regional feeder agent 79: the initial value is 0 ; the ultimate value is 1 .

Regional feeder agent 80 : the initial value is 0 ; the ultimate value is 1 .

Regional feeder agent 84: the initial value is 0 ; the ultimate value is 1 .

Fitness value is 5.60925 ; SOT is 3. 
TABLE 1: Device parameters of the SIPS.

\begin{tabular}{|c|c|c|c|}
\hline Number & Device & Power & Rank \\
\hline 1 & G1 & 21 & 0 \\
\hline 2 & G2 & 3.75 & 0 \\
\hline 3 & G3 & 3.75 & 0 \\
\hline 4 & G4 & 21 & 0 \\
\hline 5 & $\mathrm{P} 1$ & 0.005 & 0 \\
\hline 6 & M1 & 20 & 1 \\
\hline 7 & M2 & 20 & 1 \\
\hline 8 & $\mathrm{P} 2$ & 0.005 & 0 \\
\hline 9 & P3 & 0.005 & 0 \\
\hline 10 & $\mathrm{P} 4$ & 0.005 & 0 \\
\hline 11 & P5 & 0.005 & 0 \\
\hline 12 & P6 & 0.005 & 0 \\
\hline 13 & M3 & 20 & 1 \\
\hline 14 & M4 & 20 & 1 \\
\hline 15 & P7 & 0.005 & 0 \\
\hline 16 & I1 & 0.05 & 3 \\
\hline 17 & M5 & 1.2705 & 2 \\
\hline 18 & $\mathrm{I} 2$ & 1.1024 & 2 \\
\hline 19 & $\mathrm{P} 10$ & 0.005 & 0 \\
\hline 20 & I3 & 0.05 & 3 \\
\hline 21 & M6 & 0.8976 & 2 \\
\hline 22 & $\mathrm{I} 4$ & 1.4937 & 2 \\
\hline 23 & P9 & 0.005 & 0 \\
\hline 24 & I5 & 0.05 & 3 \\
\hline 25 & M7 & 1.2705 & 2 \\
\hline 26 & I6 & 0.7692 & 2 \\
\hline 27 & $\mathrm{P} 10$ & 0.005 & 0 \\
\hline 28 & I7 & 0.05 & 3 \\
\hline 29 & M8 & 1.2705 & 2 \\
\hline 30 & I8 & 3.0 & 2 \\
\hline 31 & P11 & 0.005 & 0 \\
\hline 32 & I9 & 0.05 & 3 \\
\hline 33 & M9 & 1.2705 & 2 \\
\hline 34 & $\mathrm{I} 10$ & 1.4937 & 2 \\
\hline 35 & $\mathrm{P} 12$ & 0.005 & 0 \\
\hline 36 & I11 & 0.005 & 3 \\
\hline 37 & M10 & 0.7295 & 2 \\
\hline 38 & $\mathrm{I} 12$ & 1.2705 & 2 \\
\hline 39 & $\mathrm{P} 13$ & 0.005 & 0 \\
\hline 40 & $\mathrm{I} 13$ & 0.05 & 3 \\
\hline 41 & M11 & 1.2705 & 2 \\
\hline 42 & $\mathrm{I} 14$ & 1.392 & 2 \\
\hline 43 & P14 & 0.005 & 0 \\
\hline 44 & $\mathrm{I} 15$ & 0.005 & 3 \\
\hline 45 & M12 & 0.7692 & 2 \\
\hline 46 & I16 & 1.2308 & 2 \\
\hline 47 & G5 & 21 & 0 \\
\hline 48 & G6 & 3.75 & 0 \\
\hline 49 & G7 & 3.75 & 0 \\
\hline
\end{tabular}

TABLE 1: Continued.

\begin{tabular}{|c|c|c|c|}
\hline Number & Device & Power & Rank \\
\hline 50 & G8 & 21 & 0 \\
\hline 51 & P23 & 0.005 & 0 \\
\hline 52 & M1 & 20 & 1 \\
\hline 53 & $\mathrm{M} 2$ & 20 & 1 \\
\hline 54 & P24 & 0.005 & 0 \\
\hline 55 & P25 & 0.005 & 0 \\
\hline 56 & P26 & 0.005 & 0 \\
\hline 57 & $\mathrm{P} 27$ & 0.005 & 0 \\
\hline 58 & $\mathrm{P} 28$ & 0.005 & 0 \\
\hline 59 & M3 & 20 & 1 \\
\hline 60 & M4 & 20 & 1 \\
\hline 61 & P15 & 0.005 & 0 \\
\hline 62 & I17 & 0.05 & 3 \\
\hline 63 & M5 & 1.2705 & 2 \\
\hline 64 & $\mathrm{I} 2$ & 1.1024 & 2 \\
\hline 65 & P15 & 0.005 & 0 \\
\hline 66 & $\mathrm{I} 18$ & 0.05 & 3 \\
\hline 67 & M6 & 0.8976 & 2 \\
\hline 68 & $\mathrm{I} 4$ & 1.4937 & 2 \\
\hline 69 & $\mathrm{P} 17$ & 0.005 & 0 \\
\hline 70 & I19 & 0.05 & 3 \\
\hline 71 & M7 & 1.2705 & 2 \\
\hline 72 & I6 & 0.7692 & 2 \\
\hline 73 & $\mathrm{P} 18$ & 0.005 & 0 \\
\hline 74 & $\mathrm{I} 20$ & 0.05 & 3 \\
\hline 75 & M8 & 1.2705 & 2 \\
\hline 76 & I8 & 3.0 & 2 \\
\hline 77 & P19 & 0.005 & 0 \\
\hline 78 & $\mathrm{I} 21$ & 0.05 & 3 \\
\hline 79 & M9 & 1.2705 & 2 \\
\hline 80 & $\mathrm{I} 10$ & 1.4937 & 2 \\
\hline 81 & P20 & 0.005 & 0 \\
\hline 82 & $\mathrm{I} 22$ & 0.005 & 3 \\
\hline 83 & M10 & 0.7295 & 2 \\
\hline 84 & $\mathrm{I} 12$ & 1.2705 & 2 \\
\hline 85 & $\mathrm{P} 21$ & 0.005 & 0 \\
\hline 86 & $\mathrm{I} 23$ & 0.05 & 3 \\
\hline 87 & M11 & 1.2705 & 2 \\
\hline 88 & $\mathrm{I} 14$ & 1.392 & 2 \\
\hline 89 & $\mathrm{P} 22$ & 0.005 & 0 \\
\hline 90 & $\mathrm{I} 24$ & 0.005 & 3 \\
\hline 91 & M12 & 0.7692 & 2 \\
\hline 92 & $\mathrm{I} 16$ & 1.2308 & 2 \\
\hline 93 & $\mathrm{~L} 1$ & 0.005 & 0 \\
\hline 94 & $\mathrm{~L} 2$ & 0.005 & 0 \\
\hline 95 & L3 & 0.005 & 0 \\
\hline 96 & L4 & 0.005 & 0 \\
\hline
\end{tabular}


TABLE 2: Simulation results of Example 1 compared with traditional PSO.

\begin{tabular}{|c|c|c|c|c|c|c|c|}
\hline \multirow{2}{*}{ Algorithm type } & \multicolumn{3}{|c|}{ Fitness } & \multicolumn{3}{|c|}{ SOT } & \multirow{2}{*}{ Best iteration } \\
\hline & MAX & MIN & AVG & MAX & MIN & AVG & \\
\hline Traditional PSO & 5.63215 & 5.50513 & 5.58676 & 25 & 1 & 5.6 & 27 \\
\hline MAPSO & 5.63305 & 5.50642 & 5.62084 & 22 & 1 & 3.8 & 18 \\
\hline
\end{tabular}

TABLE 3: Simulation results of Example 2 compared with traditional PSO.

\begin{tabular}{|c|c|c|c|c|c|c|c|}
\hline \multirow{2}{*}{ Algorithm type } & \multicolumn{3}{|c|}{ Fitness } & \multicolumn{3}{|c|}{ SOT } & \multirow{2}{*}{ Best iteration } \\
\hline & MAX & MIN & AVG & MAX & MIN & AVG & \\
\hline Traditional PSO & 5.60115 & 5.50582 & 5.54486 & 24 & 7 & 8.9 & 36 \\
\hline MAPSO & 5.60925 & 5.55364 & 5.59183 & 18 & 3 & 5.3 & 27 \\
\hline
\end{tabular}
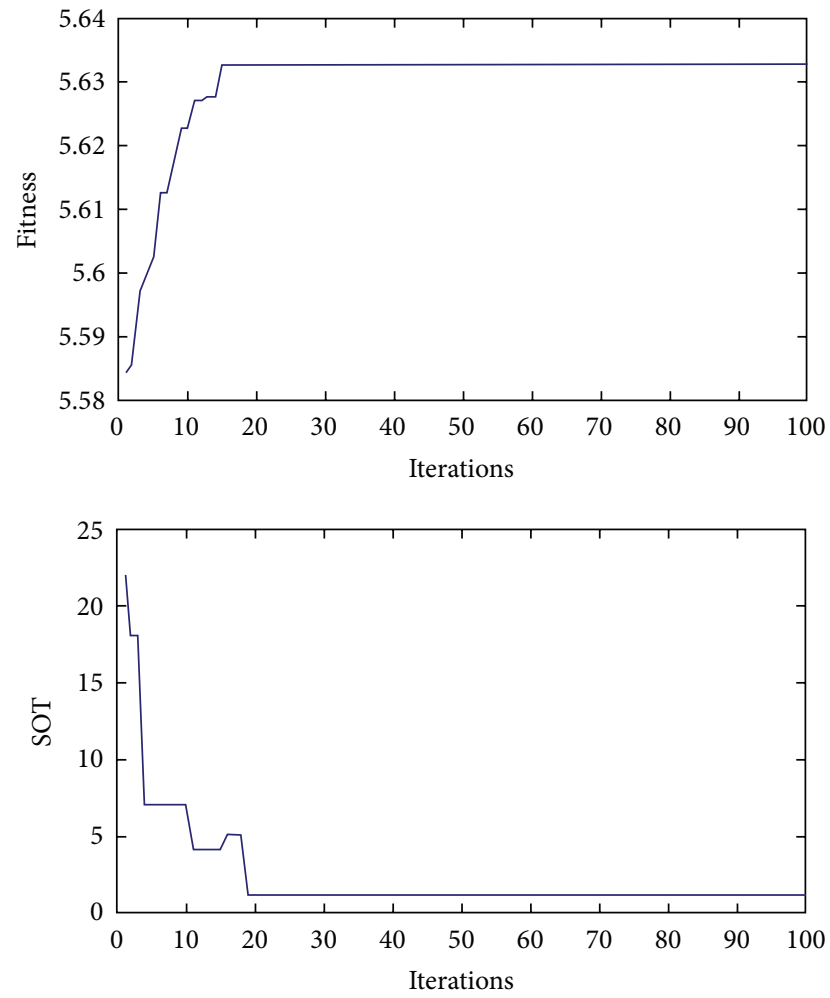

FIGURE 4: The changes of fitness and SOT of Example 1.

The changes of fitness and SOT are shown in Figure 5. When faults occur, the regional feeder agents with faults communicate with adjacent agents. The adjacent agents would firstly estimate self-capacity. If all the constraint conditions are satisfied, they would supply power to the fault agents by alternative routes. MAPSO gets the best fitness value 5.60925 from the 15th iteration, but only until the 27 th iteration it gets the minimum SOT: 3. So we get the best network reconfiguration solution from the 27 th iteration.

\section{Comparison with Traditional PSO}

In order to verify the validity of MAPSO, the simulation results of two examples are compared with traditional PSO, as shown in Tables 2 and 3.
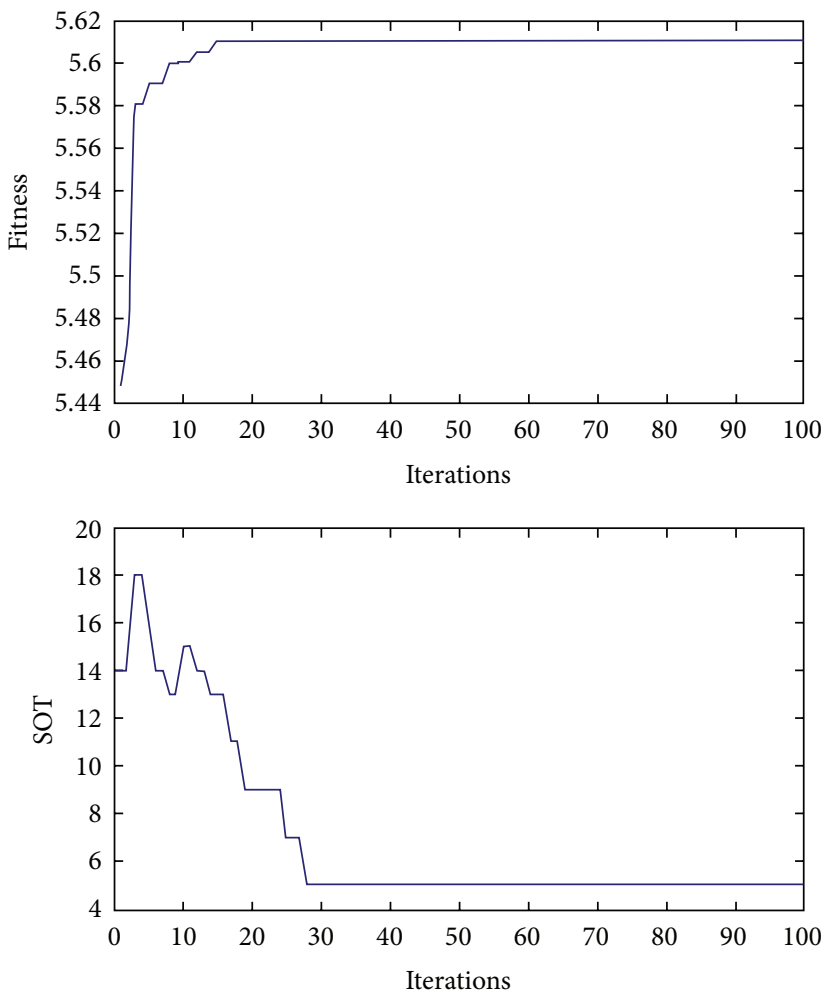

FIgURE 5: The changes of fitness and SOT of Example 2.

Tables 2 and 3 show that the application of multiagent could significantly enhance the effect of intelligent network reconfiguration. It can accelerate convergence and optimization of intelligent algorithm. In Example 1, multiagent speeds up convergence and optimization of traditional PSO. In Example 2, based on the information exchange between agents, MAPSO change the network topology in the minimum scope for reconfiguration. It gets a better reconfiguration solution.

\section{Conclusions}

The simplified network model and reconfiguration mathematical model of SIPS are established. PSO and multiagent technology are analyzed. Regional feeder agents are defined. Combining PSO with multiagent, MAPSO is presented. In 
this algorithm, regional feeder agents communicate with adjacent agents to accomplish SIPS network reconfiguration. Simulation and comparison results show that MAPSO can reconfigure SIPS network more efficient.

\section{Conflict of Interests}

The authors declare that there is no conflict of interests regarding the publication of this paper.

\section{Acknowledgment}

This work is supported by National Natural Science Foundation of China under Grant no. 51177168.

\section{References}

[1] J. Huang, X. Zhang, Y. Chen, L. Fu, and Z. Ye, "Multiobjective optimal model of service restoration for integrated ship power system and its application," Transactions of China Electrotechnical Society, vol. 25, no. 3, pp. 130-137, 2010.

[2] F. He and X. Cai, "Based on improved genetic algorithm for ship power system reconfiguration," Electrotechnical Society, vol. 21, pp. 25-30, 2006.

[3] Q. Liu, L.-B. Shi, Y.-X. Ni, and Z.-Y. Dong, "Intelligent optimization strategy of the power grid reconfiguration during power system restoration," Proceedings of the Chinese Society of Electrical Engineering, vol. 29, no. 13, pp. 8-15, 2009.

[4] H. Zhang, H. Yan, T. Liu, and Q. Chen, "Fuzzy controller design for nonlinear impulsive fuzzy systems with time delay," IEEE Transactions on Fuzzy Systems, vol. 19, no. 5, pp. 844-856, 2011.

[5] H. Zhang, H. Yan, F. Yang, and Q. Chen, "Quantized control design for impulsive fuzzy networked systems," IEEE Transactions on Fuzzy Systems, vol. 19, no. 6, pp. 1153-1162, 2011.

[6] S. K. Srivastava, K. L. Butler-Purry, and N. D. R. Sarma, "Shipboard power restored for active duty", IEEE Computer Applications in Power, vol. 15, no. 3, pp. 16-23, 2002.

[7] T. Nagata and H. Sasaki, "A multi-agent approach to power system restoration," IEEE Transactions on Power Systems, vol. 17, no. 2, pp. 457-462, 2002.

[8] H. C. Yan, Z. Z. Su, H. Zhang, and F. W. Yang, "Observerbased $H_{\infty}$ control for discrete-time stochastic systems with quantisation and random communication delays," IET Control Theory \& Applications, vol. 7, no. 3, pp. 372-379, 2013.

[9] H. Yan, H. Shi, H. Zhang, and F. Yang, "Quantized $H_{\infty}$ control for networked systems with communication constraints," Asian Journal of Control, vol. 15, no. 5, pp. 1468-1476, 2013.

[10] H. Zhang, H. Yan, F. Yang, and Q. Chen, "Distributed average filtering for sensor networks with sensor saturation," IET Control Theory \& Applications, vol. 7, no. 6, pp. 887-893, 2013.

[11] W. Zheng, X. Li, and W. Yongji, "Application of multi-agent and genetic algorithm in network reconfiguration of ship power system," Electronics \& Electrical Engineering, vol. 18, no. 9, pp. 710, 2012.

[12] F. Yushun and C. Junwei, Muti-Agnet Systems: Theory, Method and Applications, Springer Press, 2005.

[13] Z. Hongxia and Y. Quan, "Application of multi-agent technology in power system," Journal of Chongqing University, vol. 29, no. 11, pp. 53-57, 2006.
[14] H.-C. Shu, L. Tang, and J. Dong, "A survey on application of multi-agent system in power system," Power System Technology, vol. 29, no. 6, pp. 27-31, 2005.

[15] X.-F. Xie, W.-J. Zhang, and Z.-L. Yang, "Overview of particle swarm optimization," Control and Decision, vol. 18, no. 2, pp. 129-134, 2003.

[16] Z. Wang, D. Zhao, Y. Wang, and D. Liu, "Reconfiguration of shipboard power system using discrete particle swarm optimisation," International Journal of Modelling, Identification and Control, vol. 15, no. 4, pp. 277-283, 2012.

[17] J. Lu, H. Yang, and J. Du, "Improved strategy of particle swarm optimization algorithm for reactive power optimization," International Journal of Bio-Inspired Computation, vol. 2, no. 1, pp. 27-33, 2010.

[18] X. Zhang and H. Qiu, "Hybrid particle swarm optimization with $\mathrm{k}$-centres method and dynamic velocity range setting for travelling salesman problems," International Journal of BioInspired Computation, vol. 2, no. 1, pp. 34-41, 2010.

[19] J. Kennedy and R. C. Eberhart, "A discrete binary version of the particle swarm algorithm," in Proceedings of the IEEE International Conference on Systems, Man, and Cybernetics, pp. 4104-4108, IEEE Press, Piscataway, NJ, USA, October 1997.

[20] C. K. Mohan and B. Al-kazemi, "Discrete particle swarm optimization," in Proceedings of the Workshop on Particle Swarm Optimization, Purdue School of Engineering and Technology, Indianapolis, Indiana, 2001.

[21] R. Yang, H. Gao, and W. Pang, "Based on discrete particle swarm optimization algorithm for multi-user detector," Harbin Institute of Technology, vol. 37, no. 9, pp. 1303-1306, 2005.

[22] X. Wang, J. Li, and J. Xiao, "Solving the network reconfiguration of the shipboard power system DPSO greedy algorithm," Control and Decision, vol. 23, pp. 157-161, 2008. 


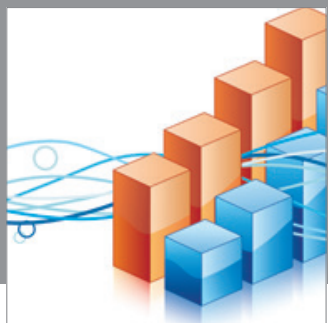

Advances in

Operations Research

mansans

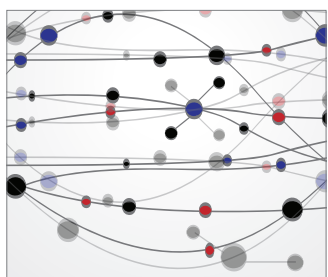

The Scientific World Journal
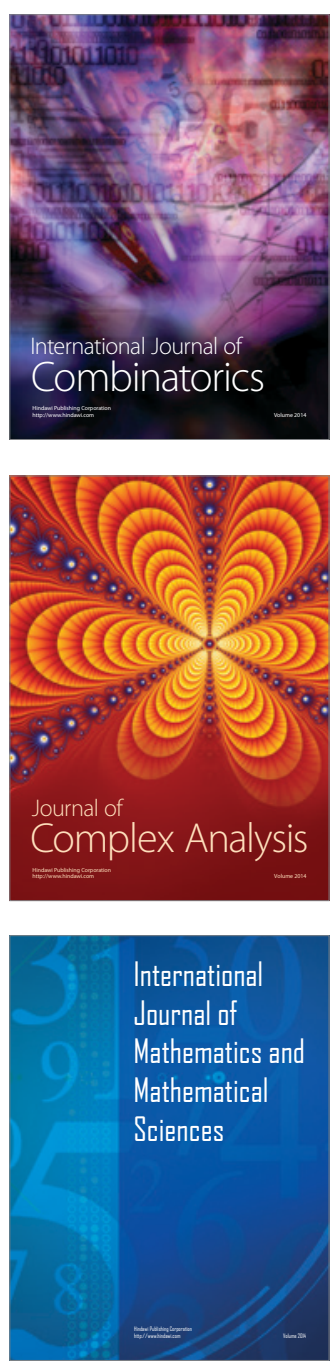
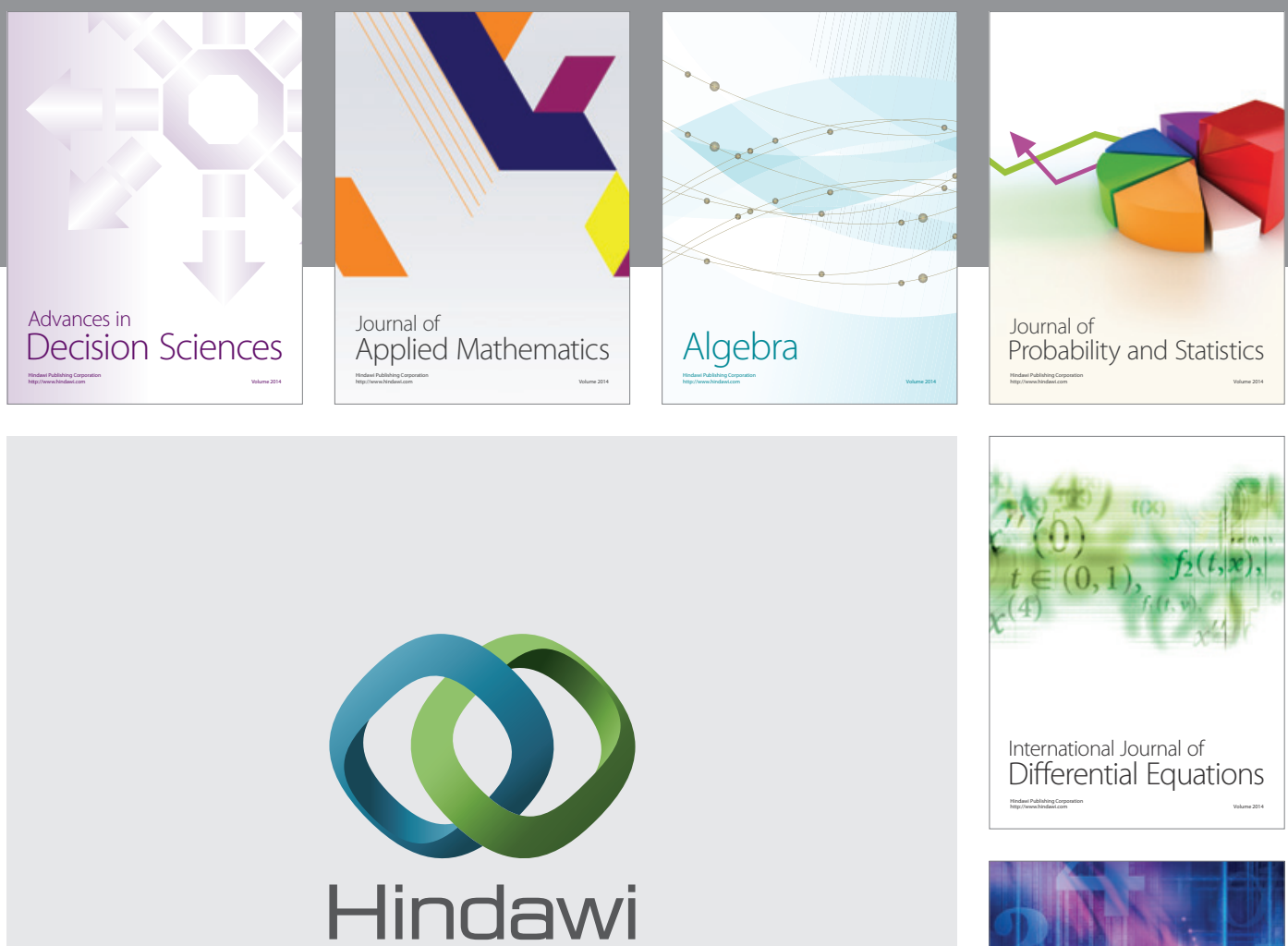

Submit your manuscripts at http://www.hindawi.com
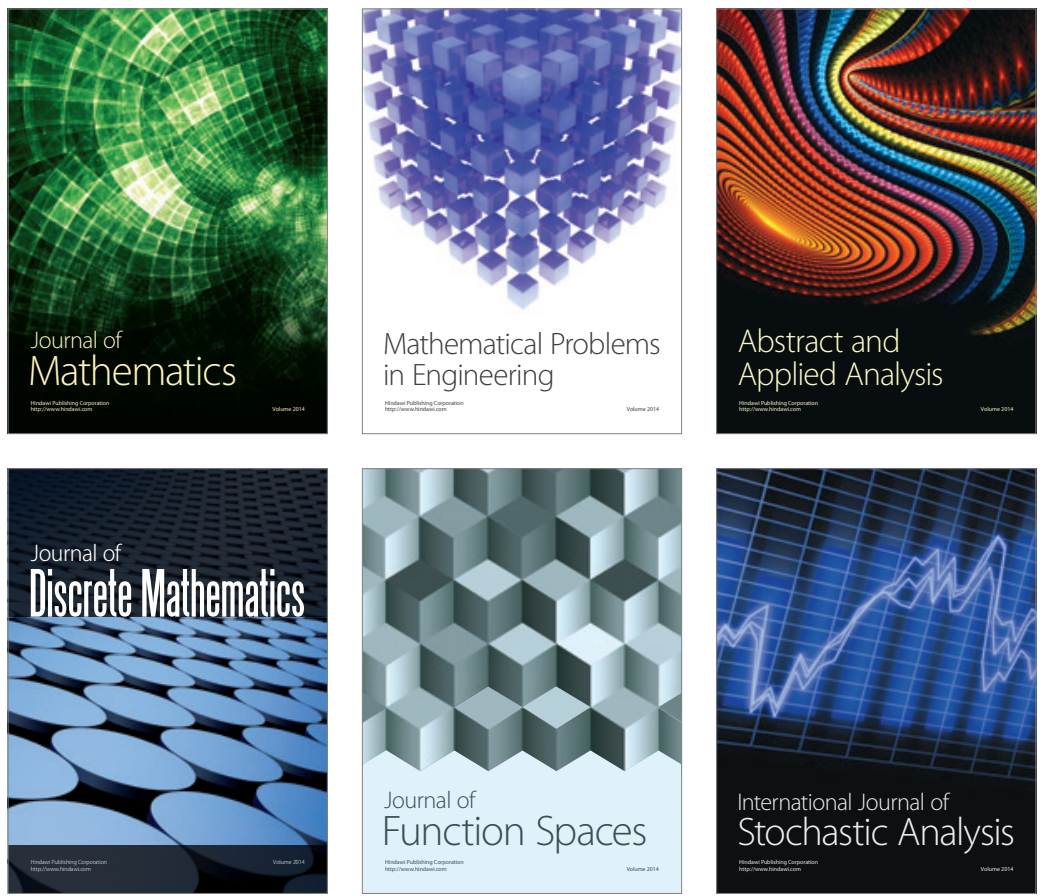

Journal of

Function Spaces

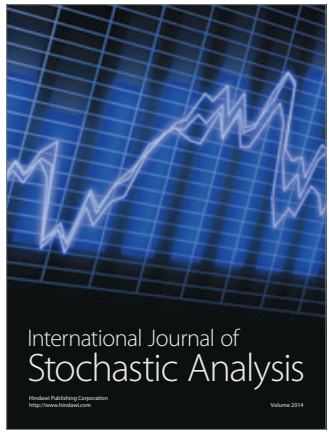

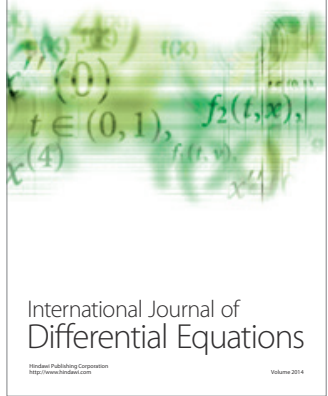
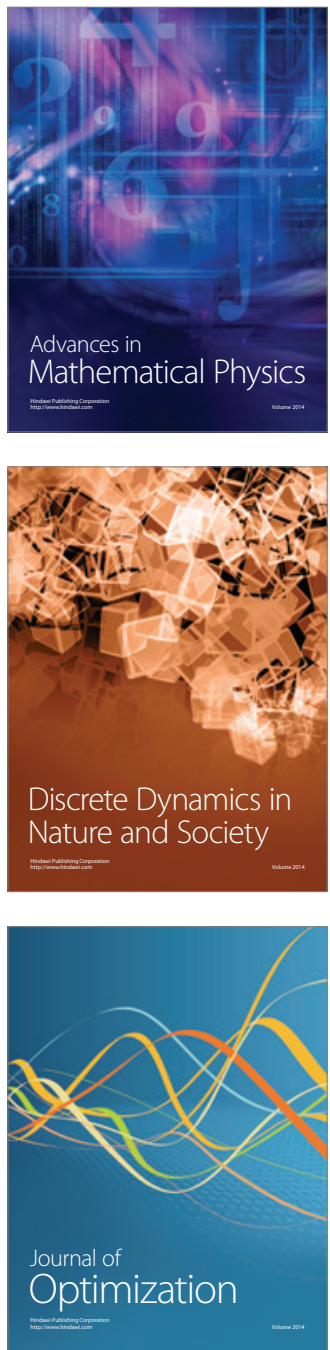